\title{
Supporting Information: The Kondo effect of a molecular tip as a magnetic sensor
}

\author{
Léo Garnier, ${ }^{\dagger}$ Benjamin Verlhac, ${ }^{*}, \dagger$ Paula Abufager, ${ }^{\ddagger}$ Nicolás Lorente, ${ }^{\top}, \S$ Maider \\ Ormaza, ${ }^{*, \dagger}$ and Laurent Limot ${ }^{*, \dagger}$ \\ $\dagger$ †niversité de Strasbourg, CNRS, IPCMS, UMR 7504, F-67000 Strasbourg, France \\ $\ddagger$ Instituto de Física de Rosario, Consejo Nacional de Investigaciones Científicas y Técnicas \\ (CONICET) and Universidad Nacional de Rosario, Av. Pellegrini 250 (2000) Rosario, \\ Argentina \\ \Centro de Física de Materiales (CFM), 20018 Donostia-San San Sebastián, Spain \\ $\S$ Donostia International Physics Center (DIPC), 20018 Donostia-San Sebastián, Spain \\ E-mail: verlhac@ipcms.unistra.fr; ormaza@ipcms.unistra.fr; limot@ipcms.unistra.fr
}

\section{Methods}

Experimental details. To carry out the measurements, we used a STM operating at $2.4 \mathrm{~K}$ in ultrahigh vacuum (base pressure below $1 \times 10^{-10} \mathrm{mbar}$ ). The $\mathrm{Cu}(100)$ surface was cleaned by repeated cycles of $\mathrm{Ar}^{+}$ion bombardment and annealing at $860 \mathrm{~K}$. The commerciallypurchased Cc molecules were sublimated in vacuo from a crucible kept at room temperature with a deposition rate of 1 monolayer/min. During deposition, the $\mathrm{Cu}(100)$ surface was held at a temperature below $150 \mathrm{~K}$. After deposition, the sample was immediately cooled down in the STM. Single Fe atoms were sublimated onto the cold $\mathrm{Cu}(100)$ surface from a $99.99 \%$ pure iron wire through an opening of the cryostat shield. During deposition, the surface temperature was $10 \mathrm{~K}$. We used a $\mathrm{W}$ tip that we cleaned in vacuo by sputter/anneal cycles. 
We then performed controlled indentations of the tip into the pristine $\mathrm{Cu}(100)$ surface to have a monoatomic tip apex, as well as a negligible electronic structure in the bias range of interest. All $d I / d V$ versus bias $(V)$ spectra were recorded via a lock-in amplifier (modulation: $200 \mu \mathrm{V}$ rms, frequency: $712 \mathrm{~Hz}$ ); the $d^{2} I / d V^{2}$ spectra were obtained by numerically deriving the $d I / d V$ spectra.

The procedure for transferring Cc from the surface to the tip is identical to the one used for nickelocene. ${ }^{1}$ The tip is positioned above the Cc with typical tunneling parameters -30 $\mathrm{mV}$ and $50 \mathrm{pA}$. Line scans across the molecule are then acquired while monitoring the line profile of Cc. To attach Cc to the tip, the tunneling bias is lowered to $-1 \mathrm{mV}$ and the current increased to $200 \mathrm{pA}$, which is equivalent to approaching the tip towards Cc by approximately 200 pm. When Cc detaches from the surface, the line profile becomes flat. The presence of $\mathrm{Cc}$ on the tip can be quickly asserted by comparing images acquired prior to and after $\mathrm{Cc}$ attachment and/or acquiring a $d I / d V$ spectrum above the copper surface.

Computational details. DFT calculations were carried out using the VASP code. $^{2,3}$ We optimized the different structures using the spin-polarized generalized gradient approximation (GGA-PBE). To improve the description of the electronic structure of the cobaltocene molecule, we used a simplified (rotationally invariant) DFT $+\mathrm{U}$ approach introduced by Dudarev and co-workers ${ }^{4}$ with an effective U-parameter $\mathrm{U}_{\text {eff }}=(\mathrm{U}-\mathrm{J})=3 \mathrm{eV}$. This value is in line with the one used in recent studies of a cobalt-based complex on $\mathrm{Cu}(111)^{5}$ where parameters $\mathrm{U}=4 \mathrm{eV}$ and $\mathrm{J}=1 \mathrm{eV}$ were employed. We used a plane-wave basis set and the projectedaugmented-wave (PAW) method $^{6}$ with an energy cutoff of $400 \mathrm{eV}$ and a $5 \times 5 \times 1 \mathrm{k}$-mesh to sample the Brillouin zone. The surface was modeled using a slab geometry with a $4 \times 4$ surface unit cell and 4 layers of metal atoms. In order to introduce long-range dispersion corrections, we employed the so-called DFT-D2 approach proposed by Grimme. ${ }^{7}$ The calculations with DFT-D3 ${ }^{8}$ and the optPBE-vdW7 $7^{9}$ non-local correlation functional indicates that the vdW scheme do not induce a strong effect neither on the adsorption nor on the magnetic properties of the system. We performed charge analysis based on the Bader ap- 
proach. ${ }^{10}$ The vibrations are computed using the finite-difference method implemented in VASP. The atoms of the tip are maintained fixed during the mode calculation, leading to a larger uncertainty in the modes at low frequencies. The simulated STM images were carried out applying the Tersoff and Hamann theory in a plane-wave basis set ${ }^{11}$ as implemented in the STMpw code. ${ }^{12}$

The Kondo density of states of spectral function is computed using the Non-Crossing Approximation (NCA) for molecular orbitals. ${ }^{13}$ The effect of vibrations is included perturbatively to all orders using the self-consistent Born Approximation. ${ }^{14}$ All integrals are performed using fast-Fourier-transform and the self-consistent iterations are stopped when the spectral function changes less than $10^{-3}$.

Cobaltocene presents a dynamical Jahn-Teller effect in the gas phase ${ }^{15}$ which must persist on the surface. As the molecular symmetry is restored by the dynamical JahnTeller effect, STM images of the molecule do not yield information on a possible Jahn-Teller distortion. Previous calculations we carried out on fullerenes using vibronic states ${ }^{16}$ showed that the experimental $d I / d V$ can be recovered in the presence of a dynamical Jahn-Teller effect by including coupled electronic and vibrational degrees of freedom. The present Kondo calculation achieves this by treating the electronic and vibrational degrees of freedom selfconsistently to all orders in the electron-vibration coupling. ${ }^{17}$ 
Table S1: Computed structural properties and magnetism for a Cc-tip in configurations 1 to 3: Energy difference $\delta E$ among tip configurations, distances between the $\mathrm{Cu}$ adatom and the nearby $\mathrm{C}$ atoms of the cyclopentadienyl ring, tilt angle of $\mathrm{Cc}$ relative to the surface normal, magnetizations of Cc and Co. The chemical accuracy of DFT was tested by using the Grimme DFT+D2 and DFT+D3 corrections, and by using the optPBE van der Waals density functional. We found the results to be robust to the method employed. The table presents the results for the Grimme DFT+D2.

\begin{tabular}{cccccc} 
Config. & $\delta E(\mathrm{eV})$ & $\mathrm{C}-\mathrm{Cu}$ distance $(\mathrm{pm})$ & angle $\left(^{\circ}\right)$ & $\left\langle S_{\mathrm{Cc}}\right\rangle\left(\mu_{\mathrm{B}}\right)$ & $\left\langle S_{\mathrm{Co}}\right\rangle\left(\mu_{\mathrm{B}}\right)$ \\
\hline \hline $\mathbf{1}$ & 0 & $211,230,288,302,327$ & 16 & 0.89 & 0.83 \\
\hline $\mathbf{2}$ & 0.13 & $251,206,260,310,306$ & 4 & 0.88 & 0.82 \\
\hline $\mathbf{3}$ & 0.18 & $216,215,285,287,316$ & 16 & 0.91 & 0.85 \\
\hline
\end{tabular}



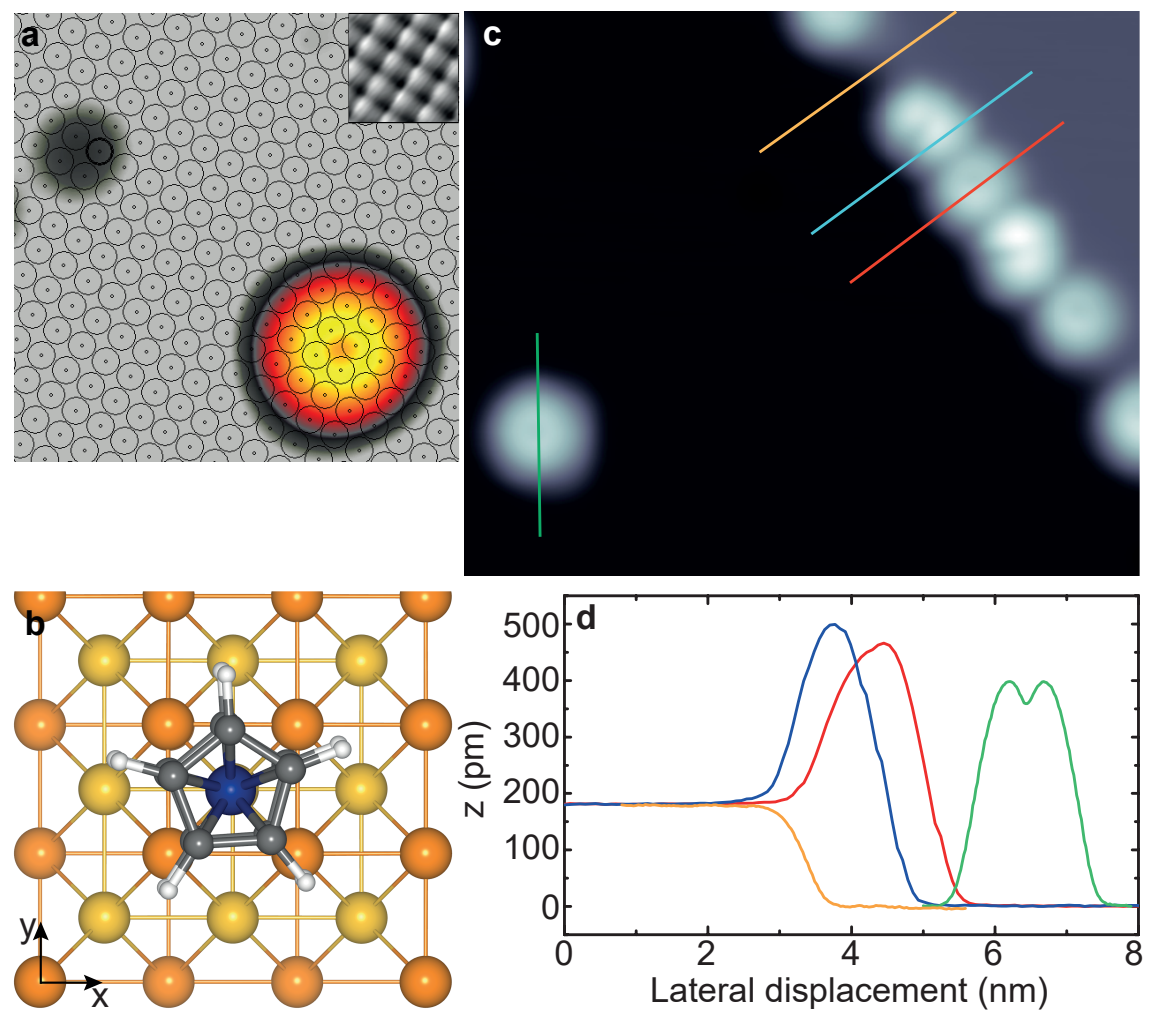

Figure S1: (a) Cobaltocene on a $\mathrm{Cu}(100)$ terrace co-adsorbed with a Fe atom $\left(4 \times 4 \mathrm{~nm}^{2}\right.$, $-70 \mathrm{mV}, 20 \mathrm{pA}$ ). To determine the adsorption site of $\mathrm{Cc}$, we imaged the corrugation of $\mathrm{Cu}(100)$ through a tip-assisted manipulation of the Fe atom (inset: $1 \times 1 \mathrm{~nm}^{2}, 7 \mathrm{mV}$, $280 \mathrm{nA}) .{ }^{18} \mathrm{~A}$ grid representing the $\mathrm{Cu}(100)$ surface was simulated and positioned onto the image of panel (a) using the Fe atoms as markers. (b) Cobaltocene adsorption site as determined by DFT. The energetic analysis was performed by relaxing Cc in three highlysymmetric sites (Cp ring centered on top, bridge and hollow sites). (c) Cobaltocene at the step edge of $\mathrm{Cu}(100)$ (size: $12 \times 8 \mathrm{~nm}^{2}$, sample bias: $20 \mathrm{mV}$, tunneling current: $30 \mathrm{pA}$ ) and, (d) Line profiles of two cobaltocene molecules at the step (red and blue solid lines), of the pristine $\mathrm{Cu}$ step (solid orange line), and of cobaltocene on the copper terrace (solid green line). The difference in height between a $\mathrm{Cc}$ adsorbed at a step edge and on a terrace is $35 \mathrm{pm}$, which is smaller than the step height of copper $(180 \mathrm{pm})$. This indicates that Cc adsorbs on the bottom edge of the step and is likely tilted. The image (and $d I / d V$, see main text) resemblance to a Cc-tip hints to a cobaltocene interacting with a single edge atom. 

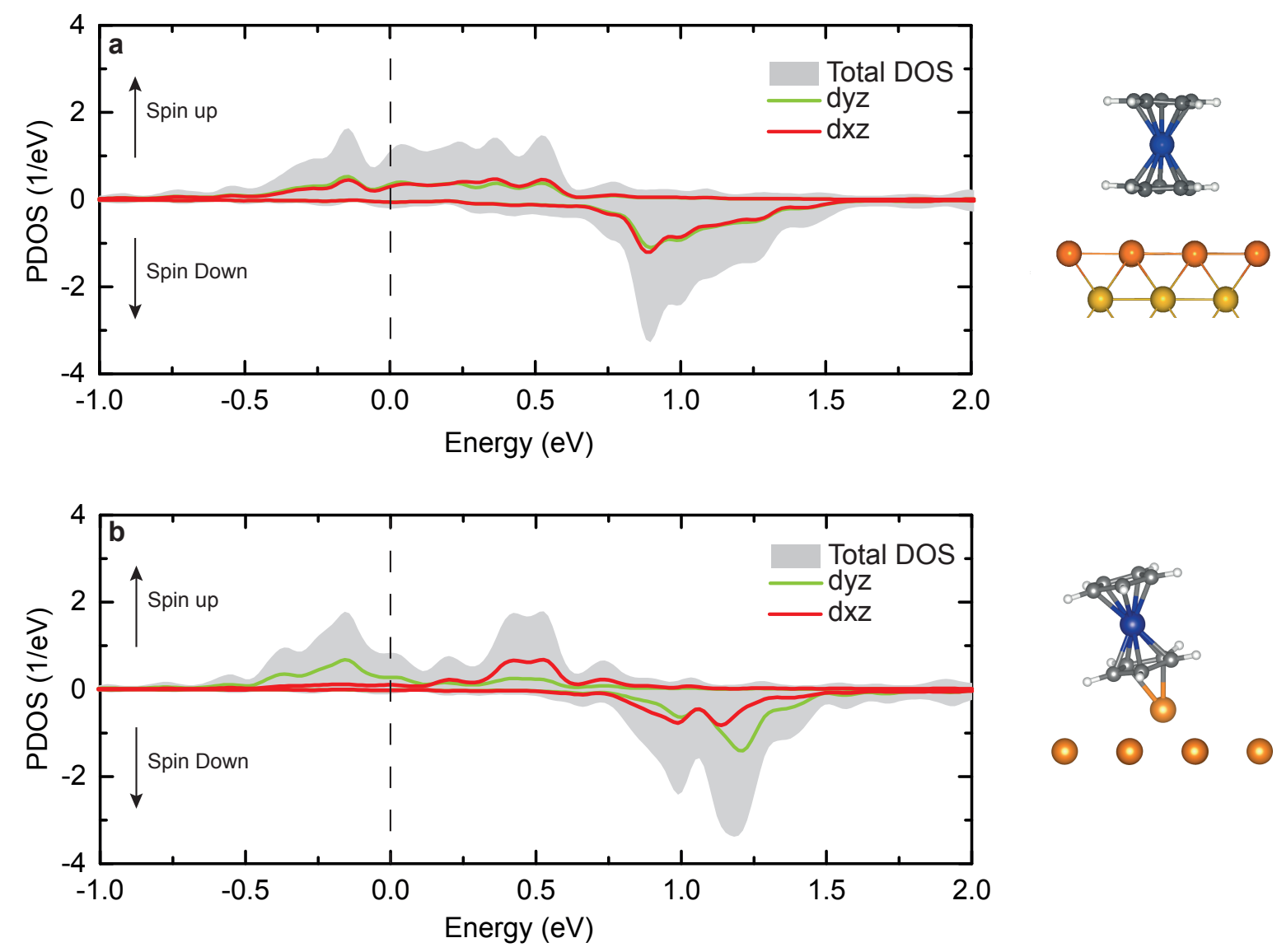

Figure S2: (a) Projected density of states (PDOS) for Cc when adsorbed on $\mathrm{Cu}(100)$ in the relaxed configuration of Figures 1d,e, and (b) for the Cc-tip in configuration 1. The solid red and green lines correspond to the projection onto the $\operatorname{Co}\left(d_{x z}\right)$ and the $\operatorname{Co}\left(d_{y z}\right)$ atomic orbitals, respectively, while the total PDOS into Co, C, H is in light grey. Top panel: spin-up PDOS, bottom panel: spin-down PDOS. 


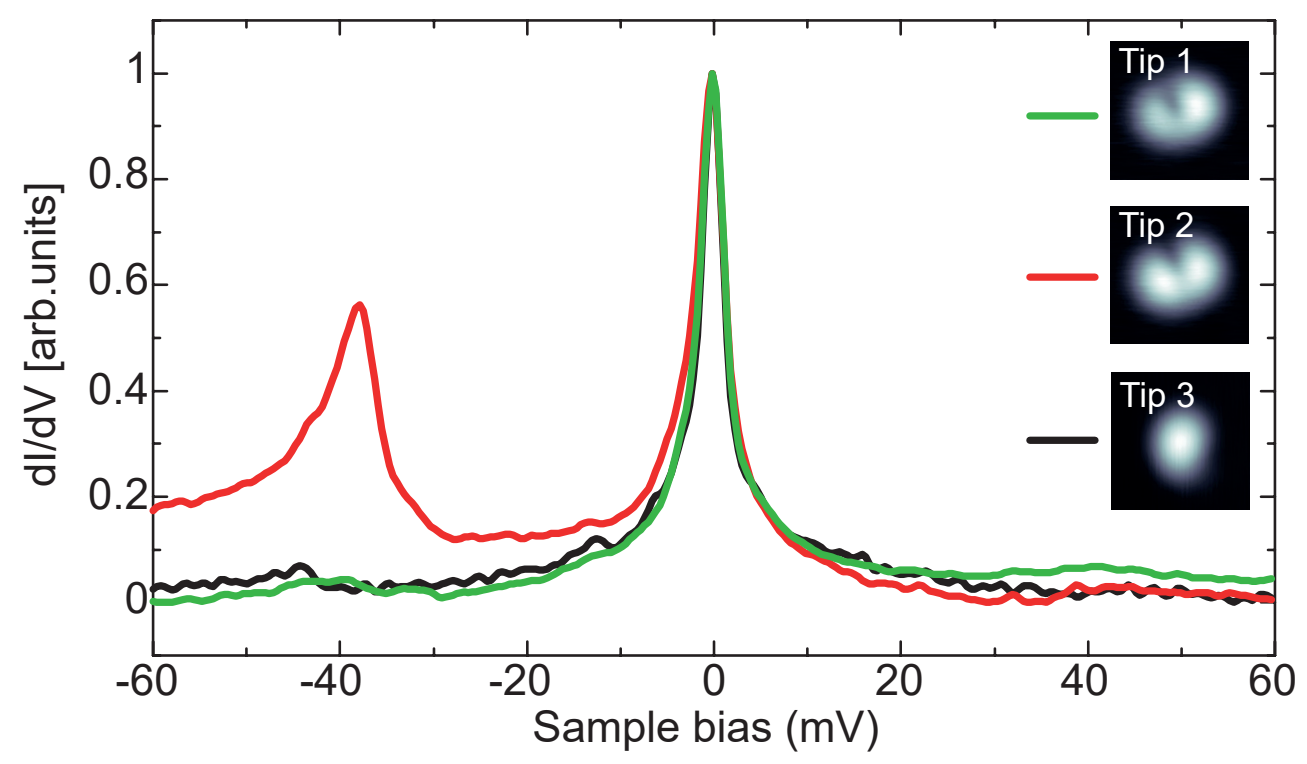

Figure S3: $d I / d V$ spectra acquired above a Fe atom on $\mathrm{Cu}(100)$ using Cc-tips in configuration 1, and 2 and $\mathbf{3}$ (sample bias:-60 mV, tunneling current: $100 \mathrm{pA}$ ). A Frota fit using Eq. (1) yields a $\Gamma$ of $0.76,0.88$ and $0.68 \mathrm{meV}$, respectively, which emphasizes that the Kondo resonance is similar for the three tip configurations. The vibrational Kondo peaks show, instead, tip dependency, in particular their amplitude. Note that, unlike the spectra of Figures 2d,e that were acquired above the copper surface, here the relative position of the Cc-tip to the Fe atom can impact the intensity of the satellite peaks. A similar dependency is observed in the spectra acquired with a sharp metal tip above a cobaltocene on a step edge (Figures S7b,c). 

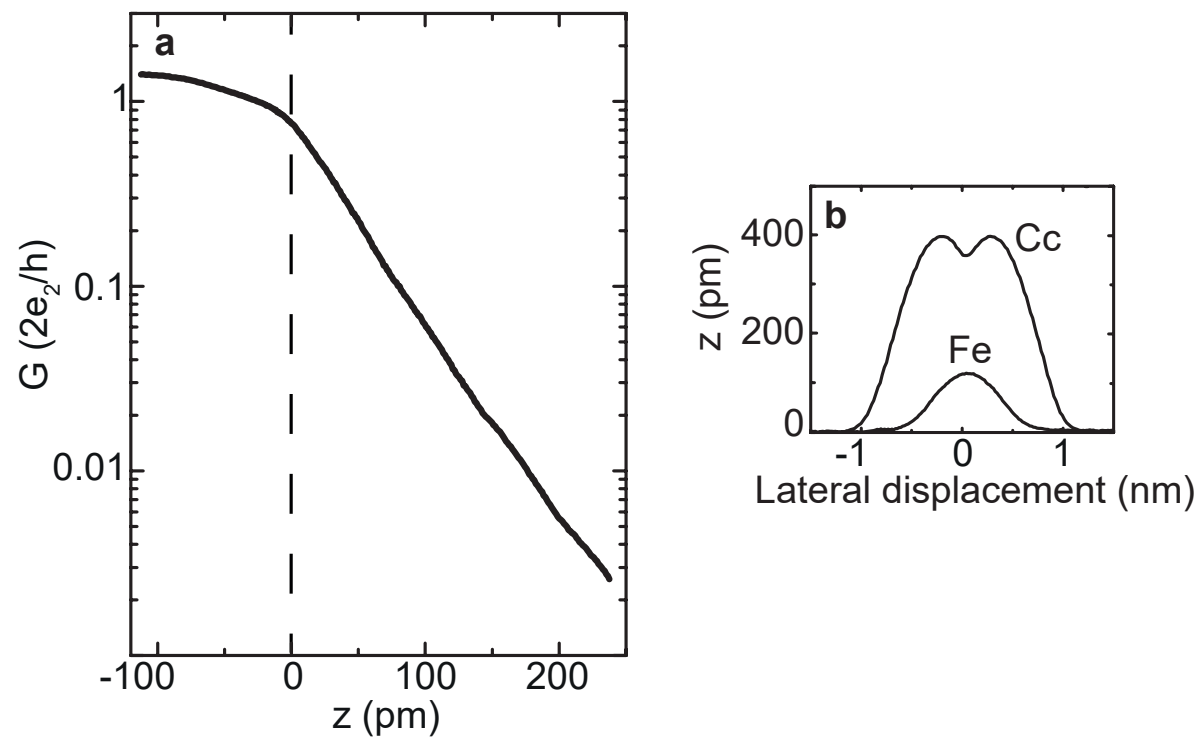

Figure S4: (a) Current-versus-tip-displacement curve acquired at bias of $V=-5 \mathrm{mV}$ with a Cc-tip above the $\mathrm{Cu}(100)$ surface. The contact distance $(z=0)$ is identified trough a change in slope of the trace. ${ }^{19}$ Upon retracting the tip, cobaltocene remains attached to the tip apex so that the procedure can be repeated several times if necessary. (b) Line profiles of Cc on a terrace and of a Fe atom. To determine the distance between the Cc-tip and the Fe atom, we subtract the apparent height of iron on the surface $(h=115 \mathrm{pm}$ at $V=-5 \mathrm{mV})$ from the contact distance determined previously on copper: $z(\mathrm{Fe})=z-h$. The distance cannot be determined directly through a point contact of the Cc-tip to iron as contact formation is unstable. 


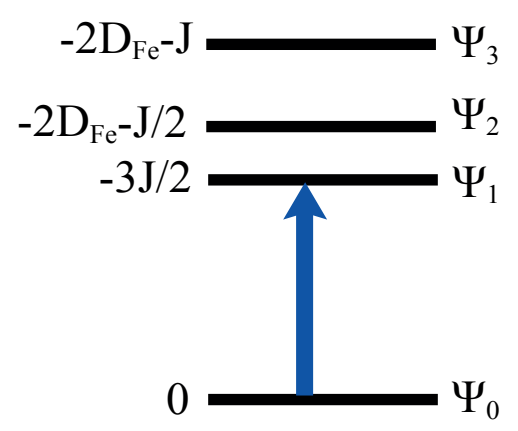

\begin{tabular}{|l|l|}
\hline$\Psi_{3}$ & $\mathrm{~m}_{\mathrm{Cc}}= \pm 1 / 2, \mathrm{~m}_{\mathrm{Fe}}= \pm 1 / 2$ \\
\hline$\Psi_{2}$ & $\mathrm{~m}_{\mathrm{Cc}}=\mp 1 / 2, \mathrm{~m}_{\mathrm{Fe}}= \pm 1 / 2$ \\
\hline$\Psi_{1}$ & $\mathrm{~m}_{\mathrm{Cc}}= \pm 1 / 2, \mathrm{~m}_{\mathrm{Fe}}= \pm 3 / 2$ \\
\hline$\Psi_{0}$ & $\mathrm{~m}_{\mathrm{Cc}}=\mp 1 / 2, \mathrm{~m}_{\mathrm{Fe}}= \pm 3 / 2$ \\
\hline
\end{tabular}

Figure S5: Spin-state diagram for a cobaltocene exchange coupled to a Fe atom on $\mathrm{Cu}(100)$ with $D_{\mathrm{Fe}}=-0.85 \mathrm{meV}$ and $J=-1.3 \mathrm{meV}$ (antiferromagnetic coupling). We have taken $S_{\mathrm{Cc}}=1 / 2$ and $S_{\mathrm{Fe}}=3 / 2$. The blue arrow indicates the spin excitation associated to the Cc spectrum.

\section{Model spin Hamiltonian for the Cc-tip above a Fe atom}

The interaction between the Cc-tip and the Fe atom on the surface was modeled using the simplified Hamiltonian $\hat{H}=-g \mu_{\mathrm{B}} B_{\mathrm{ex}} \hat{S}_{z}$ as displayed in Eq. (3) of the main text. This Hamiltonian can be deduced from the more general Hamiltonian:

$$
H=D_{\mathrm{Fe}} \hat{S}_{z, \mathrm{Fe}}^{2}-J \hat{S}_{z, \mathrm{Cc}} \cdot \hat{S}_{z, \mathrm{Fe}} .
$$

We take the $z$-axis along the out-of-surface direction neglecting the small tilt of the Cc molecule (see discussion below). The first term accounts for any possible anisotropy of the Fe adatom on $\mathrm{Cu}(100)$. The Fe atom can possesses only axial out-of-plane anisotropy since it is adsorbed on the hollow site of a fourfold symmetric $\mathrm{Cu}(100)$ surface. The sign and strength of $D_{\mathrm{Fe}}$ is, however, still unknown, but we note that the magnetic anisotropy for Fe on a $\mathrm{Cu}(111)$ is $D_{\mathrm{Fe}}=(-0.85 \pm 0.2) \mathrm{meV} \cdot{ }^{20,21}$ The second term of the equation accounts for the coupling between the two spin systems. We suppose that the two spins interact via an antiferromagnetic Ising-like coupling as observed in previous work on the nickelocene tip. ${ }^{22}$ The spin-state diagram and the eigenfunctions of the Cc-Fe system are sketched in Figure S5. The eigenfunctions of the system, $\left|\psi_{i}\right\rangle(i=0,1,2,3)$, are a linear combination 
of $\left|m_{\mathrm{Cc}} ; m_{\mathrm{Fe}}\right\rangle$. The only spin excitation detected is $\psi_{0} \rightarrow \psi_{1}$ and involves a spin-flip of the Cc molecule. Excitation $\psi_{0} \rightarrow \psi_{2}$ involves a change of spin momentum for the Fe atom, but does not lead to distinguishable spectroscopic features in the $d I / d V$ due to strong screening and broadening effects induced by the metal surface. ${ }^{20}$ The third excitation, $\psi_{0} \rightarrow \psi_{3}$, is forbidden since it does not conserve total spin momentum.

The ground state $\left|\psi_{0}\right\rangle$ is a doublet state and corresponds to an antiferromagnetic configuration where the Fe spin is anti-aligned with the Cc spin along the $z$-axis. The excited state $\left|\psi_{1}\right\rangle$ corresponds to the ferromagnetic configuration. In the manuscript, for clarity, we limit our analysis to a $\left|m_{\mathrm{Fe}}=-3 / 2\right\rangle$ spin without loss of generality. This enables to simplify Equation (S1) by neglecting the anisotropy term of Fe and reformulating the interaction term as $J \hat{S}_{z, \mathrm{Fe}} \hat{S}_{z, \mathrm{Cc}}=g \mu_{B} B_{\mathrm{ex}} \hat{S}_{z, \mathrm{Cc}}$.

In the discussion above, we neglected the tilt angle of Cc relative to the $z$-axis ( $\theta$ hereafter). The strength of the magnetic interaction is determined by the hybridization between the $\mathrm{Cc}$ and Fe orbitals, which depends on the $\mathrm{Fe}-\mathrm{Cc}$ distance $(z)$ and on the angle between orbitals, hence on $\theta$. The exchange field is then $B_{\mathrm{ex}}=|J(z, \theta)|\left\langle S_{\mathrm{z}, \mathrm{Fe}}\right\rangle / g \mu_{\mathrm{B}}$. In the manuscript we show that $J$ depends exponentially on $z$, which is typical for an exchange interaction. We may then assume that $\theta$ does not change with $z$, hence it enters in our analysis as a fixed parameter. We can therefore neglect the tilt angle, as the only effect it will have is to change the value of $J$. In practice, it is difficult to anticipate how $J$ will change with $\theta$ as this depends on the nature of the magnetic coupling, which in the present case is a mixture of a direct and a superexchange interaction. A comparative study among the different Cc-tip configurations could eventually clarify the role of $\theta$. 


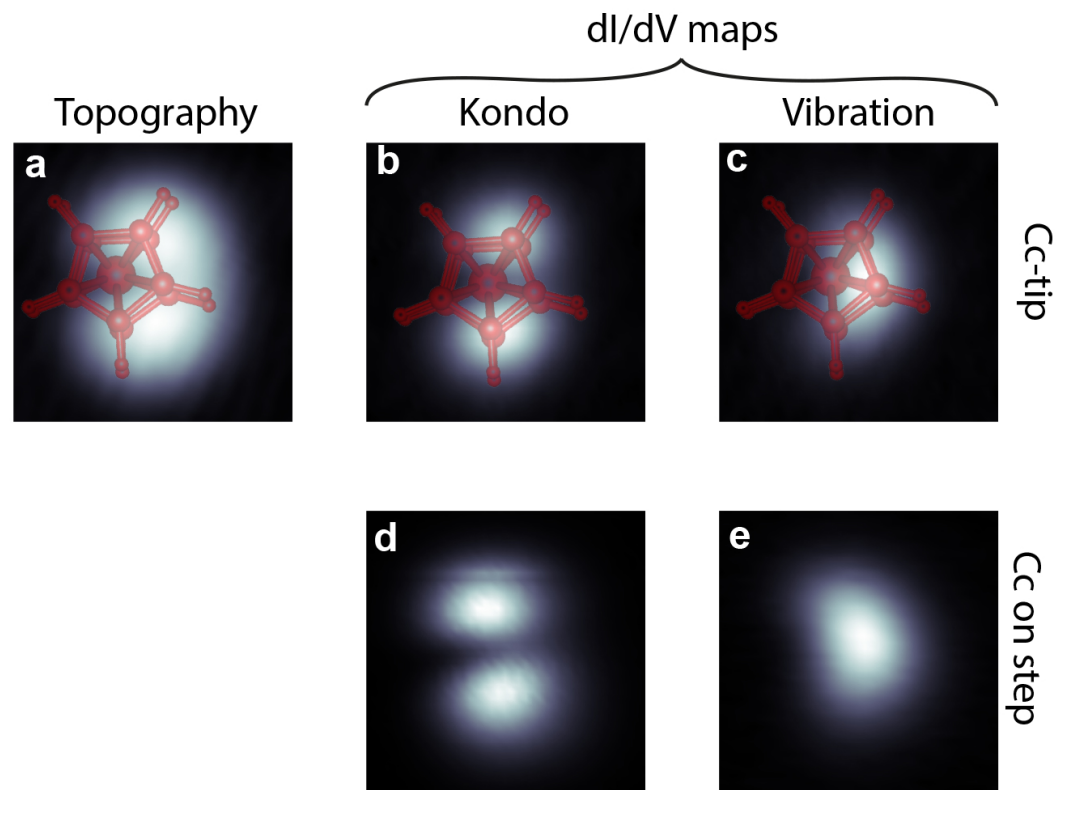

Figure S6: (a) Fe atom adsorbed on $\mathrm{Cu}(100)$ imaged with Cc-tip 2 (image size: $1.6 \times 1.6 \mathrm{~nm}^{2}$, sample bias: $60 \mathrm{mV}$, tunneling current: $30 \mathrm{pA}$ ), and constant-height $d I / d V$ map of the same area acquired with (b) $-2 \mathrm{mV}$, (c) $-38 \mathrm{mV}$. The constant-height maps reflect the spatial distribution of the Kondo resonance and of the main vibrational Kondo peak, respectively. (d) Constant-height $d I / d V$ map of Cc adsorbed on a $\mathrm{Cu}(100)$ step $\left(1.6 \times 1.6 \mathrm{~nm}^{2}, 30 \mathrm{mV}\right.$, $30 \mathrm{pA}$ ) acquired with $+4 \mathrm{mV}$ and (e) with $+45 \mathrm{mV}$ - keep in mind that the bias polarity is reversed in this case compared to panels (b) and (c). All constant-height images were acquired at a tip-molecule distance of approximately $0.4 \mathrm{~nm}$. The chemical structure of cobaltocene is superimposed to the images in (a-c) as a guide to the eye. While the Kondo resonance matches the two-lobe pattern of the SOMO, the vibrational peak is compatible with a tilted cyclopentadienyl ring. 


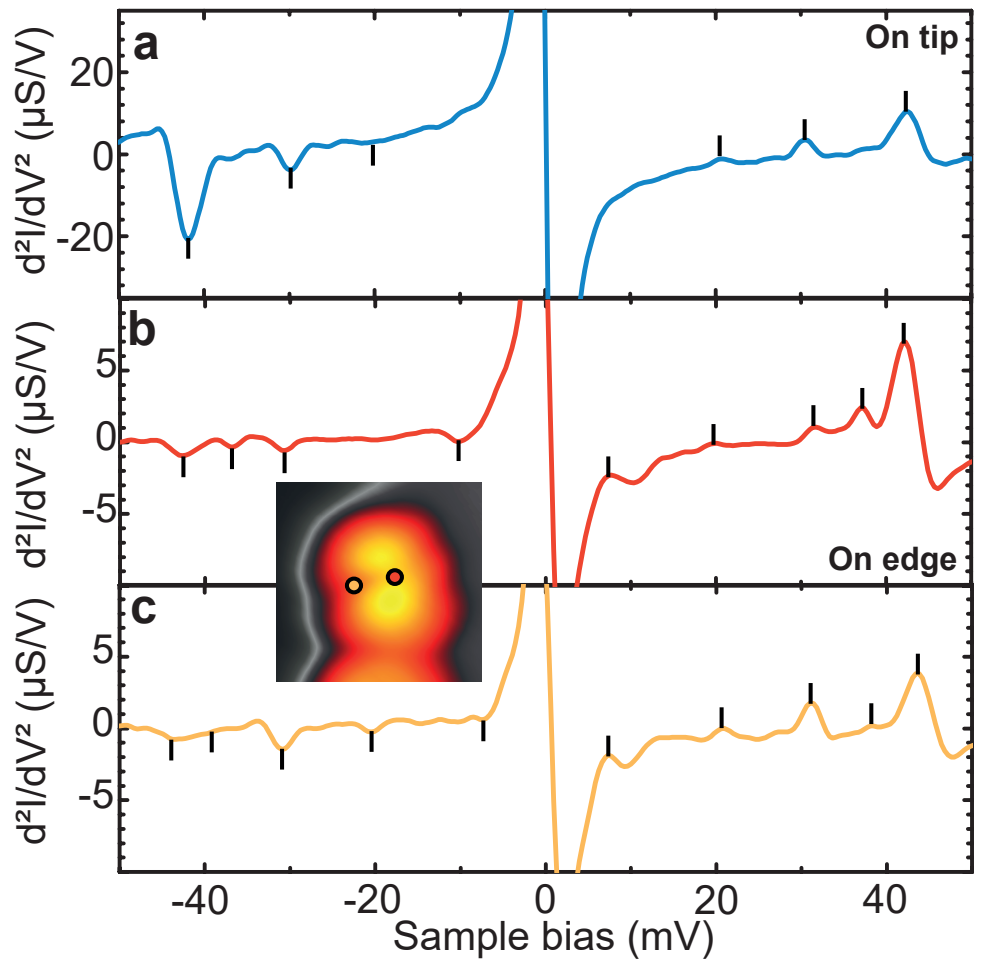

Figure S7: (a) $d^{2} I / d V^{2}$ spectrum of a Cc-tip in configuration 2 (feedback loop opened at $-70 \mathrm{mV}$ and $2 \mathrm{nA}$ ). (b-c) $d^{2} I / d V^{2}$ spectra for a Cc at a step edge ( $-75 \mathrm{mV}$ and $\left.200 \mathrm{pA}\right)$. The spectrum in (b) and (c) where acquired by positioning the $\mathrm{Cu}$-terminated tip at two locations of $\mathrm{Cc}$, which are marked, respectively, by a red and yellow dot in the image of panel (b). The peak positions are marked by a vertical line in the spectra. Their position, which is symmetric with bias polarity, shows negligible dependence on Cc adsorption or intramolecular position contrary to the peak amplitude. 

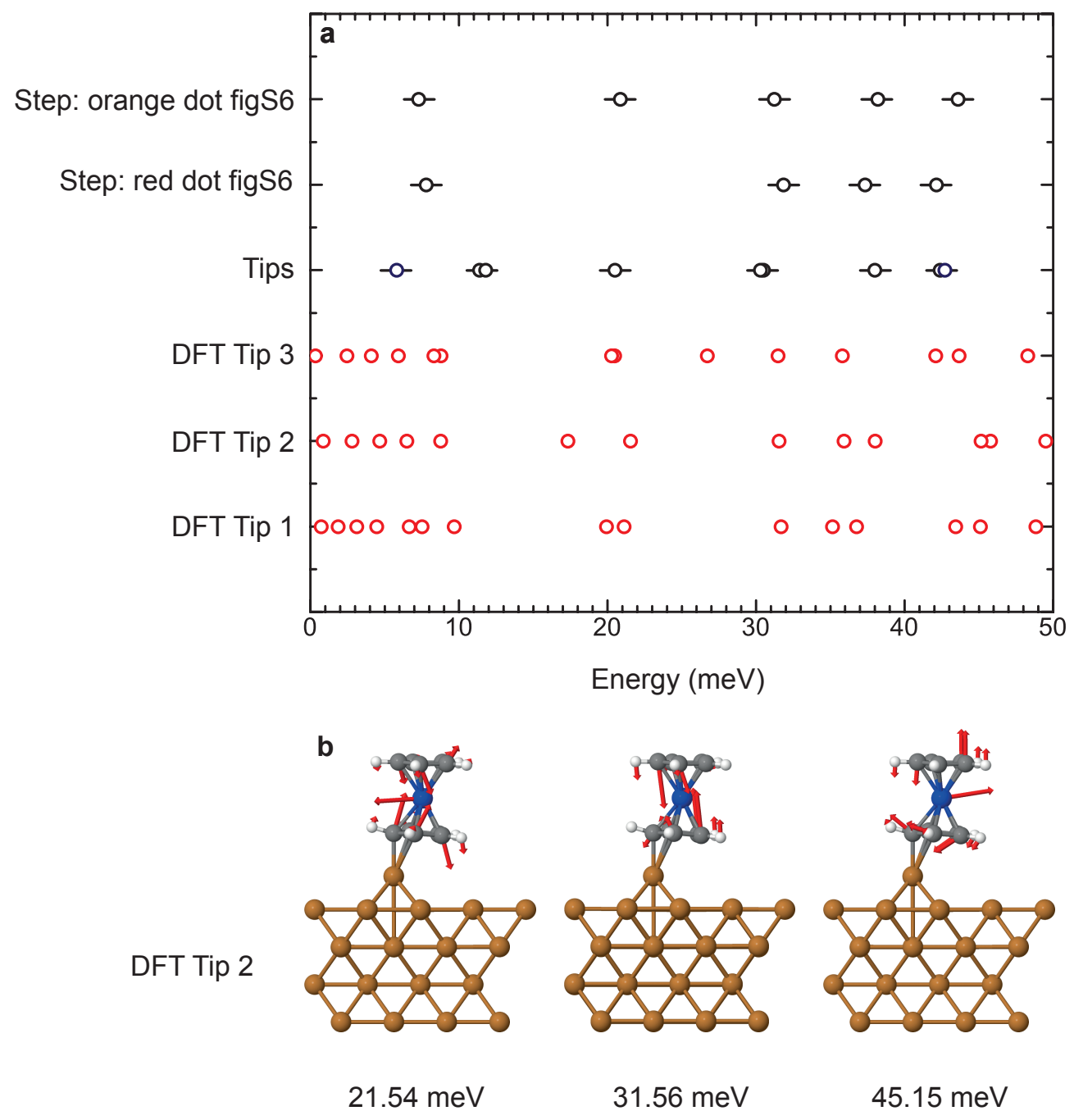

Figure S8: (a) Experimental (black dots) and DFT-computed (red dots) vibrational energies. The experimental energies comprise those of the Cc-tip (all configurations) and of Cc at the step edge (positions marked by a red and orange dot in Figure S6, respectively). The DFTcomputed energies correspond to configurations 1 to 3 of Figure 2b. Please note that the Kondo resonance impairs the observation of modes having energy $<5 \mathrm{meV}$. Some modes may also not be observable due to a weak electron-vibration coupling, which depends on molecular configuration on the surface/tip and how it is addressed. (b) Calculated vibrational modes for Cc-tip 2. 


\section{References}

(1) Ormaza, M.; Bachellier, N.; Faraggi, M. N.; Verlhac, B.; Abufager, P.; Ohresser, P.; Joly, L.; Romeo, M.; Scheurer, F.; Bocquet, M.-L.; Lorente, N.; Limot, L. Efficient SpinFlip Excitation of a Nickelocene Molecule. Nano Lett. 2017, 17, 1877-1882, PMID: 28199115.

(2) Kresse, G.; Furthmüller, J. Efficiency of Ab-Initio Total Energy Calculations for Metals and Semiconductors Using a Plane-Wave Basis Set. Comput. Mater. Sci. 1996, 6, 15.

(3) Kresse, G.; Furthmüller, J. Efficient Iterative Schemes for Ab Initio Total-Energy Calculations Using a Plane-Wave Basis Set. Phys. Rev. B 1996, 54, 11169.

(4) Dudarev, S. L.; Botton, G. A.; Savrasov, S. Y.; Humphreys, C. J.; Sutton, A. P. Electron-energy-loss spectra and the structural stability of nickel oxide: An LSDA+U study. Phys. Rev. B 1998, 57, 1505-1509.

(5) Knaak, T.; Gruber, M.; Lindström, C.; Bocquet, M.-L.; Heck, J.; Berndt, R. LigandInduced Energy Shift and Localization of Kondo Resonances in Cobalt-Based Complexes on $\mathrm{Cu}(111)$. Nano Lett. 2017, 17, 7146-7151.

(6) Kresse, G.; Joubert, D. From Ultrasoft Pseudopotentials to the Projector AugmentedWave Method. Phys. Rev. B 1999, 59, 1758.

(7) Grimme, S. Semiempirical GGA-Type Density Functional Constructed with a LongRange Dispersion Correction. J. Comput. Chem. 2006, 27, 1787.

(8) Grimme, S.; Antony, J.; Ehrlich, S.; Krieg, H. A consistent and accurate ab initio parametrization of density functional dispersion correction (DFT-D) for the 94 elements H-Pu. J. Chem. Phys. 2010, 132, 154104.

(9) Klimeš, J. c. v.; Bowler, D. R.; Michaelides, A. Van der Waals density functionals applied to solids. Phys. Rev. B 2011, 83, 195131. 
(10) Henkelman, G.; Arnaldsson, A.; Jónsson, H. A fast and robust algorithm for Bader decomposition of charge density. Comput. Mater. Sci 2006, 36, $354-360$.

(11) Bocquet, M.-L.; Lesnard, H.; Monturet, S.; Lorente, N. In Computational Methods in Catalysis and Materials Science; Santen, R. A. v., Sautet, P., Eds.; Wiley-VCH Verlag GmbH \& Co. KGaA, 2009; pp 199-219.

(12) Lorente, N.; Robles, R. STMpw. 2019; https://zenodo.org/record/3581159.

(13) Korytr, R.; Lorente, N. Multi-orbital non-crossing approximation from maximally localized Wannier functions: the Kondo signature of copper phthalocyanine on $\mathrm{Ag}(100)$. J. Phys. Condens. Matter 2011, 23, 355009.

(14) Roura-Bas, P.; Tosi, L.; Aligia, A. A. Replicas of the Kondo peak due to electronvibration interaction in molecular transport properties. Phys. Rev. B 2016, 93.

(15) Zlatar, M.; Schlpfer, C.-W.; Fowe, E. P.; Daul, C. A. Density functional theory study of the Jahn-Teller effect in cobaltocene. Pure Appl. Chem. 31 Jul. 2009, 81, 1397 1411.

(16) Frederiksen, T.; Franke, K. J.; Arnau, A.; Schulze, G.; Pascual, J. I.; Lorente, N. Dynamic Jahn-Teller effect in electronic transport through single $\mathrm{C}_{60}$ molecules. Phys. Rev. B 2008, 78, 233401.

(17) Roura-Bas, P.; Tosi, L.; Aligia, A. A. Nonequilibrium transport through magnetic vibrating molecules. Phys. Rev. B 2013, 87, 195136.

(18) Heinrich, B. W.; Iacovita, C.; Brumme, T.; Choi, D.-J.; Limot, L.; Rastei, M. V.; Hofer, W. A.; Kortus, J.; Bucher, J.-P. Direct Observation of the Tunneling Channels of a Chemisorbed Molecule. J. Phys. Chem. Lett. 2010, 1, 1517-1523.

(19) Limot, L.; Kröger, J.; Berndt, R.; Garcia-Lekue, A.; Hofer, W. A. Atom Transfer and Single-Adatom Contacts. Phys. Rev. Lett. 2005, 94, 126102. 
(20) Khajetoorians, A. A.; Lounis, S.; Chilian, B.; Costa, A. T.; Zhou, L.; Mills, D. L.; Wiebe, J.; Wiesendanger, R. Itinerant Nature of Atom-Magnetization Excitation by Tunneling Electrons. Phys. Rev. Lett. 2011, 106, 037205.

(21) Pacchioni, G. E.; Gragnaniello, L.; Donati, F.; Pivetta, M.; Autès, G.; Yazyev, O. V.; Rusponi, S.; Brune, H. Multiplet features and magnetic properties of Fe on $\mathrm{Cu}(111)$ : From single atoms to small clusters. Phys. Rev. B 2015, 91, 235426.

(22) Verlhac, B.; Bachellier, N.; Garnier, L.; Ormaza, M.; Abufager, P.; Robles, R.; Bocquet, M.-L.; Ternes, M.; Lorente, N.; Limot, L. Atomic-scale spin sensing with a single molecule at the apex of a scanning tunneling microscope. Science 2019, 366, 623-627. 